\title{
On the Grammatical Status of Le in Mandarin
}

\author{
Man Yuan \\ Department of Foreign Languages, Yinxing Hospitality Management College, Chengdu University of Information Technology, Chengdu, \\ China
}

\section{Email address: \\ 77673817@qq.com}

\section{To cite this article:}

Man Yuan. On the Grammatical Status of Le in Mandarin. International Journal of Language and Linguistics. Vol. 6, No. 5, 2018 , pp. $148-153$. doi: $10.11648 /$ j.ijll.20180605.12

Received: July 31, 2018; Accepted: September 19, 2018; Published: October 10, 2018

\begin{abstract}
Le is a functional morpheme in Mandarin, which can appear in two places, immediately after the verb (verb-le) or in a sentence-final position (sentence-le). Traditionally, verb-le is often referred to as a perfective aspect marker denoting completion, while sentence-le is generally considered as a sentence final particle which signals a change-of-state meaning. Based on Smith's aspect theory, which calls the grammatical aspect the viewpoint aspect and the lexical aspect the situation aspect, this paper argues that both les are perfective aspect markers derived from one super-le. Besides, it also compares le with guo, which is another post-verbal perfective aspect marker whose aspectual status has been well-established in the literature, and points out that guo differs from verb-le in that it always denotes the completion of event. It is further argued that the reason why le conveys distinct meanings lies in its position in the sentence. And thus, the conclusion is drawn as: although verb-le and sentence-le denote different meanings, they are essentially the same in terms of the three following aspects: (1) Both of them are perfective aspect viewpoint markers. (2) They have the same temporal interpretations. (3) They show the same variance from guo. Therefore, there is just one le which is a perfective aspect marker. If it is placed after the verb, it will have the terminative reading. If it is in the sentence-final position, it will form a Perfect and have a change-of-state reading. Given such difference, a syntactic representation of le in the clausal structure is proposed, in which verb-le is generated in the AspP lower within vP while sentence-le resides in the AspP adjoined to TP. The reason why guo cannot occur with verb-le is the different aspectual meanings they convey. Guo is concerned with the experience the subject has while sentence-le is about the state change.
\end{abstract}

Keywords: Chinese Le, Functional Morpheme, Mandarin

\section{Introduction}

Le is a functional morpheme in Mandarin Chinese. It can be found in two places, either immediately after the verb or at the very end of a sentence as in (1).

(1) wo mai-le shu

I buy-LE book

'I bought a book.'

(2) wo mai shu le.

I buy book LE.

'I have bought a book.'

Traditionally, they are called verb-le and sentence-le respectively. Although numerous studies have been done on them, there is still no agreement on their exact nature and status. There are two broad views. One view is that there is just one-le, with verb-le and sentence-le as its variants [12]. The other view is that verb-le and sentence-le are simply homophones with different meanings and functions $[1,6,12]$. Generally speaking, the second view gets upper hand in the literature. The main argument for this dichotomy lies in the semantic differences between the sentences with verb-le and sentence-le respectively. The most popular view is that verb-le is a perfective aspect marker denoting completion of an event [6]. Under this assumption, (1) has the meaning that the event of buying a book was completed. On the other hand, sentence-le is often regarded as a sentence-final particle denoting a change-of-state meaning [3]. That is, a new state denoted by the sentence has begun and formed a contrast with the state before [12]. Therefore, the meaning of (2) is that the state that I have bought a book has begun and there is a contrast between the state of having a book now and that of not having a book before.

In this paper, I side with the one-le camp and argue that both verb-le and sentence-le are perfective aspect markers derived from one super-le. This paper is organized as follows. First, I 
present a theory of aspect in which the study is framed. Then I discuss the syntax and semantics of verb-le and sentence-le respectively. Afterwards, I compare them with another Mandarin perfective marker guo. In the end I discuss their positions in Mandarin clausal structure.

\section{The Theory of Aspect}

Since le is traditionally associated with the aspect system of Mandarin, it is necessary to establish the framework of aspect in order to evaluate or discuss its grammatical status. As reviewed by $\mathrm{Li}$, most theories of aspect recognized two kind of aspect, i.e. grammatical and lexical [7]. Smith calls the former viewpoint aspect and the latter situation aspect [11]. To simplify the issue, I adopt Smith's classification in this paper.

According to Smith, situation aspect is concerned with classification of the situations represented in verb constellations in terms of their temporal properties [11]. She considers three temporal parameters, i.e. dynamism, durativity and telicity, and differentiates five situation types: State, Activity, Accomplishment, Semelfactive and Achievement. States are durative and stative with no inherent endpoints, such as 'know somebody', 'be sick'. Activities are dynamic, durative but not telic, such as 'walk in the park', 'swim'. They don't encode a natural final endpoint, or in Smith words, they have an arbitrary final endpoint. Accomplishments are dynamic, durative and telic, such as 'walk to school', 'write a letter'. Therefore, they have an inherent final endpoint. Semelfactives are dynamic but not telic or durative, such as 'jump', 'knock'. They only consist of a single stage of event and hence are inherently bounded. Achievements are dynamic, telic but not durative, such as 'reach New York', 'break the window'. Like Semelfactives, they also represent a single-stage event but with a resultative reading.

Smith demonstrates that the relationship between certain verb-constellations and situation types does not always hold [11]. With some additional linguistic forms such as adverbials, situation types can be changed. For example, in (3a), the verb 'jump' is Semelfactive. However, because the adverbial 'for an hour' has a durative meaning, the whole sentence is derived as an Activity. Similarly, (3b) has a shifted stative value because of the adverbial 'every day'.

(3) John jumped for an hour.

b. John walked to school every day.

Viewpoint aspect, according to Smith, gives temporal perspective to a sentence and is usually marked by a grammatical morpheme [11]. She classifies it into three categories: perfective, imperfective and neutral. Perfective viewpoint "focuses a situation in its entirety, including both initial and final endpoints" as in sentence (4). Imperfective viewpoint focuses "part of a situation, including neither initial nor final endpoints" as in sentence (5). Neutral viewpoint is aspectually vague in that sentences with it lack perfective or imperfective aspect markers and thus are neither perfective nor imperfective. English doesn't have neutral viewpoint, but other languages such as French, Chinese do.

(4) John read a book.
(5) John was reading a book.

Another concept relevant to the study is the Perfect construction as in (6).

(6) Now John has arrived. [11]

b. Last Sunday John had already arrived.

c. Next Sunday John will have already arrived.

According to Smith, Perfect sentences have four interrelated properties. First, the event represented in the sentence happens before Reference Time (RT). The RTs in the three sentences in (6) are the present, the past and the future respectively. Second, the sentence has a resultative stative reading. For example, (6a) implies a state of affairs that John is here as a result of the event of arriving. Third, it usually has a closed viewpoint aspect, i.e. perfective viewpoint, as illustrated in (6). Finally, the subject has a property which results from participation in the event denoted by the sentence. Thus, in (6a) John has the property of being here.

\section{The Aspectual Role of Le}

\subsection{Verb-Le}

Verb-le is generally regarded as a perfective aspect marker without too much controversy. One of its striking properties is that it cannot co-occur with the imperfective viewpoint morpheme zai as in (7a).

(7) a. wo xiawu zai kan (*le) shu.

I afternoon ZAI read (LE) book

('I was reading a book in the afternoon.')

b. wo xiawu kan-le shu.

'I read a book in the afternoon.'

Zai denotes a progressive meaning as reflected in the intended translation of (7a). The focus of the sentence is not on the entirety of the event of reading a book but rather an arbitrary internal phase. In such case, le cannot occur. By contrast, as (7b) shows, the same situation is interpreted with a non-imperfective viewpoint with only le. The focus is shifted to the event in its entirety. This suggests that verb-le has the property of a perfective viewpoint marker.

This characteristic is further evidenced in the following sentences.

(8) women gang da-le yijia. (Activity)

we just fight-LE one.fight

'We just fought.'

(9) wo zuotian xie-le yifengxin. (Accomplishment)

I yesterday write-LE one.letter

'I wrote a letter yesterday.'

(10) wo qiao-LE yixia men. (Semelfactive)

I knock-LE one.time door

'I knocked at the door.'

(11) wo xiawu daoda-le mudidi. (Achievement)

I afternoon arrive-LE destination

'I arrived at the destination in the afternoon.'

As the above sentences display, le can occur in the four situations of Activity, Accomplishment, Semelfactive and Achievement. The focus of the situation is on the entirety of the event and a meaning of completion is conveyed. Besides 
past tense, it can also occur in sentences with future tense as in (12).

(12) wo mai-le shu jiu qu

I buy-LE book then go

'I will go as soon as I buy a book.'

(12) denotes two events which have not happened yet: going and buying a book. Obviously, there is a temporal sequence between the two events. That is, buying a book will happen before going. Therefore, even though the event of buying a book has not occurred, it must be completed for the other event of going to happen. In other words, le can also convey completion in the future tense. Thus, verb-le contributes the perfective viewpoint aspect to the sentence.

However, it cannot occur in States as in (13).

(13) a. wo shi *le daxuesheng.

I be LE college.student

('I am a college student.')

b. ta renwei *le wo shi daxuesheng.

he think LE I be college.student

('He thinks that I am a college student.')

c. wo jingchang mai-*le shu.

I often buy-LE book

('I often buy books.')

As $(13 a, b)$ show, it cannot co-occur with stative verb constellations at the basic level. In (13c), although mai shu 'buy book' is an Accomplishment, the adverbial jingchang 'often' imposes a habitual reading on the sentence and transforms it into a State. In this case, it's ungrammatical for verb-le to appear. This restriction is expected if verb-le is a perfect aspect marker. As introduced in section 2, perfective viewpoint focuses on a bounded event with initial and final endpoints. Since States don't have inherent endpoints, they are naturally incompatible with perfective viewpoint. ${ }^{1}$

It should be noted that while verb-le denotes the meaning of completion in the situation types of Activity, Semelfactive and Achievement it does not necessarily imply it in Accomplishments [11].

(14) \#women gang da-le yijia, dan mei da wan.

we just fight-LE one.fight but not fight finish

('We were just fighting, but didn't finish it.')

(15) wo zuotian xie-le yifengxin, dan mei write finish

I yesterday write-LE one.letter but not xie wan

'I was writing a letter yesterday but didn't complete the task.'

(9) wo zuotian xie-le yifengxin.

I yesterday write-LE one.letter

'I wrote a letter yesterday (and finished it).'

As (14) shows, the conjunction is contradictory, which suggests that the event of fighting must be completed. However, sentence (15) shows that the event of writing a letter may not be finished. Therefore, the meaning of completion is not semantically denoted in sentence (9). Its inherent meaning

1 This is different from English perfective system in that States can have perfective viewpoint in English. Smith holds that it is the default or unmarked property of perfective viewpoint that it is incompatible with States [11]. Thus, English is a marked language in terms of this. should be the termination of the event. ${ }^{2}$

This contrast is interesting in that Accomplishments have an inherent closed reading with initial and final endpoints. Therefore, in perfective viewpoint, they should denote completion as argued by Smith [11]. However, as can be seen here, this is not necessarily true. But whether it demotes termination or completion, it is undeniable that verb-le represents the perfective viewpoint, for after all in the temporal schema there is a bounded event with initial endpoint and final endpoint. It's just that for some sentences the final endpoint is arbitrary as in the case of 'xie xin' while for some others it is the natural endpoint.

\subsection{Sentence-le}

Sentence-le is usually treated as a sentence-final article with special discoursal or pragmatic meaning [3]. However it also has syntactic-semantic functions. Consider (16) and (17) from Zhu [15]. As the contrast them shows, the function of sentence-le is not just as simple as denoting speaker's attitude. Only with the addition of sentence-le in (17), the inherent meaning of (16) has changed. Thus, sentence-le must play a greater role than a sentence-final particle.

(16) wo zai zhe-er zhu-le wu nian.

I at here live-LE five years

'I lived here for five years.' (i.e. 'I am not living here now')

(17) wo zai zhe-er zhu wu nian le.

'I have lived here for five years.' (i.e. 'I am still living here now')

A close examination of its distribution reveals that it can occur in all the situation types, as displayed in the following examples.

(18) women gang da-(le) yijia le. (Activity)

we just fight-(LE) one.fight LE

'We have just fought.'

(cf. 'I didn't fight before.')

(19) wo gei ta xie-(le) xin le. (Accomplishment)

I to he write-(LE) letter LE

'I have written him a letter'

(cf. 'I didn't write a letter to him before.')

(20) wo qiao-(LE) yixia men le. (Semelfactive)

I knock-(LE) one.time door LE

'I have knocked at the door.'

(cf. 'I didn't knock before')

(21) wo daoda-(le) mudidi le. (Achievement)

I arrive-LE destination LE

'I arrived at the destination in the afternoon.'

2 It should be noted that it is not the case that le cannot denote completion in all Accomplishments. Consider (i).

(i) \#wo zuotian ban-le suoyou-de shu, dan mei ban wan.

I yesterday move-le all-DE book but not carry finish.

('I moved all the books yesterday, but didn't finish.')

This conjunction is contradictory, suggesting that the event of moving ten books is completed. This difference from (15) may have something to do with the object. In (i), the object is definite and countable noun. Therefore, if something has been done on them, the event must have been completed. However, in (15), the object has an abstract reading (note 'write a letter' is different from 'tear a letter'). Therefore, the event can have the possibility of not being completed. 
(cf. 'I was not in the destination before.')

(22) a. wo shi (*le) daxuesheng le. (State)

I be LE college.student LE

'I have become a college student now.'

(cf. 'I was not a college student before.')

b. wo zhidao yuanyin le.

I know reason LE.

'I have come to know the reason now.'

(cf. 'I didn't know the reason before.')

In all these sentences, sentence-le signals that the state of affairs denoted by the sentence holds now. It conveys a change-of-state meaning and there is a comparison between the state in the past and at present. Chao says that this is an indication of inchoativity [1]. That is, a new state has begun. This is indeed true. But looked at another way, it also can be interpreted as a result. The two concepts are not contradictory in that a new state must be the result of some event or action. Besides, it is also noted in the examples that sentence-le and verb-le can co-occur. But the meaning is still the same as when there is no verb-le. In other words, verb-le is overridden by sentence-le.

The properties of the examples above indicate that they are Perfect constructions. First, the situation precedes Reference time which is the present in the examples. This is obvious in all the sentences except (22). On the surface, the situation parallels the Reference Time. But the event denoted by the sentence has already happened or been realized before the sentence is spoken. Otherwise there would be no change of state. So, when someone says 'wo shi daxuesheng le', he or she must have been already been a college student, or else the new state that he or she is college student cannot begin. Second, as argued above, they have a resultative stative reading. Third, the subject has a special property as a result of change of state. For instance, in (21), 'women' has the new property of being in the destination.

This observation is also evidenced by the fact that sentence-le cannot occur in sentences with a habitual reading such as (23).

(23) wo jingchang mai shu *le.

I often buy book LE

('I often buy books.')

In (23), the adverbial 'jingchang' denotes a habit of buying a book. Even though it is stative, it is not resultative. Therefore it is not a Perfect sentence. The fact that sentence-le cannot occur in this context suggests that it is an indispensible part of Perfect construction. Moreover, the contrast between the sentences with sentence-le as in (18) to (22) and the sentences without sentence-le as in (8) to (11) demonstrates that it is sentence-le that makes the sentence a Perfect. Since Perfect sentences have perfective viewpoint, it is reasonable to assume that sentence-le contributes this function. In other words, sentence-le is also a perfective viewpoint marker.

Comparing verb-le and sentence-le, it can also be noted that sentence-le does not necessarily denote the meaning of completion of an event in Accomplishment as in (24).

(24) wo gei ta xie xin le, dan mei xie wan.

I to him write letter LE but not write finish
'I have written him a letter, but have not finished.'

This comparison implies that verb-le and sentence-le denotes the same temporal meaning. The difference is that sentence-le can also form a Perfect.

\subsection{Difference from Guo}

Guo is another post-verbal perfective aspect marker whose aspectual status has been well-established in the literature. According to Smith, it presents a closed situation in the past and there is no discontinuity with the present [11]. Besides, it has an experiential meaning so that it emphasizes the experience the subject had in the past. So the sentence in (25) means that they have the experience of going to the USA but are no longer there.

(25) tamen qu-guo meiguo.

they go-GUO USA

'They once went to the USA.'

Guo differs from verb-le in that it always denotes the completion of event. So in (26a), the event of writing a letter must have been completed, as indicated by the contradiction in (26b).
(26)
a. wo gei ta xie-guo xin

I to he write-GUO letter

I once wrote him a letter.

b. \# wo gei ta xie-guo xin, dan mei xie wan.

I to he write-GUO letter but not write finish

('I once wrote him a letter but didn't finish it.')

However, as can be seen in (15) repeated here, verb-le does not necessarily imply completion of event.

(15) wo zuotian xie-le yifengxin, danshi mei write finish

I yesterday write-LE one.letter but not xie wan

'I was writing a letter yesterday but didn't complete the task.'

This contrast is borne out by the experiential meaning of guo which presents a discontinuity from the present. Consider one more example.

(27) tamen qu-le meiguo.

they go-LE USA

'They have gone to the US' (i.e.)

(27) is different from (25) because it has the meaning that they are still in the USA. Since their temporal meanings are different, guo and le cannot co-occur after verbs as in the following. ${ }^{3}$

(28) tamen qu-guo-le meiguo

(29) wo kan-guo-le zheben xiaoshuo.

I see-GUO-LE this novel

'I once saw the novel.'

(30) tamen qu-le meiguo.

3 Native speakers have different responses as to the degree of ungrammaticality of (28) and (29). Some feel they are absolutely ungrammatical, while some report that they are odd but still acceptable. Despite the controversy, it is certain that they are not natural. This is further evidenced by the observation that if an interjection such ' $a$ ' is inserted between le and the complement, the sentences will be grammatical as in (ii) and (iii). In this case, 'meiguo' and 'zheben xiaoshuo' function as an additional remark and le should be interpreted as sentence-le instead of verb-le.

(ii) tamen qu-guo-le a, meiguo

(iii) wo kan-guo-le a, zheben xiaoshuo 
they go-LE USA

'They have been to the USA.'

(31)

tamen qu-guo meiguo.

they go-GUO USA

'They once went to the USA.'

Guo is also different from sentence-le, though both of them can form Perfect constructions. The most striking variation lies in the meaning of the Perfect they form. Consider the contrast between (30) and (25).

As explained above, (25) with guo means that they are not in the USA now. By contrast, (30) with sentence-le implies that they are still there. Such contrast is expected due to the different meanings of the two kinds of Perfect constructions. The one with guo emphasizes the experience the subject had in the past which is irrelevant to the present moment, while the one with sentence-le conveys a change-of-state meaning which holds now. Accordingly, their temporal meanings are also different, as verb-le differs from guo.

Given their different temporal meanings, guo generally cannot co-occur with sentence-le either. Therefore in (31c), when guo and sentence-le both appear, the sentence is ungrammatical.

(32) a. wo zai meiguo kanjian guo ta.

I in USA see GUO him

'I once saw him in the USA.'

b. wo zai meiguo kanjian ta le

'I have seen him in the USA.'

c. * wo zai meiguo kanjian guo ta le

However, the issue is complicated by (32).

(33) a. wo gei ta xie-guo xin.

I to he write-(LE) letter LE

'I once wrote him a letter.'

b. wo gei ta xie xin le.

'I have just written him a letter.'

c. wo gei ta xie-guo xin le.

'I have just written him a letter.'

(33c) shows that the sentence is right with both guo and sentence-le. However, the meaning of guo is different in that it does not have an experiential reading. Rather, as indicated by the English translation, it simply denotes that 'I have just finished the event of writing him a letter'. This is further evidenced by the fact that the adverbial 'cengjing' which implies past experience cannot occur in (33c) but can occur in (33a).

(34) a. wo cengjing gei ta xie-guo xin.

'I once wrote him a letter.'

b. * wo cengjing gei ta xie-guo xin le.

$\mathrm{Hu}$ and Fan argues that there are two guos [4]. One is the experiential guo as presented in this part. The other guo is similar to verb-le. Along this assumption, the guo in (33c) can be interpreted as the second guo. Therefore, it can be replaced by verb-le without change of meaning as in (34).

(35) wo gei ta xie-le xin le.

'I have just written him a letter.'

Summarizing, verb-le and sentence-le are different guo. However, the differences are the same. In the first place, the temporal meaning of guo is different from that of either le.
Besides, both les cannot co-occur with guo.

\section{A unified Account of Le}

On the basis of the analysis above, it can be concluded that although verb-le and sentence-le denote different meanings, they are essentially the same. First, both of them are perfective aspect viewpoint markers. Second, they have the same temporal interpretations. Third, they show the same variance from guo. Therefore, there is just one le which is a perfective aspect marker. The reason why it conveys distinct meanings lies in its position in the sentence. If it is placed after the verb, it will have the terminative reading. If it is in the sentence-final position, it will form a Perfect and have a change-of-state reading. Given such difference, I propose the following syntactic representation of le in the clausal structure.

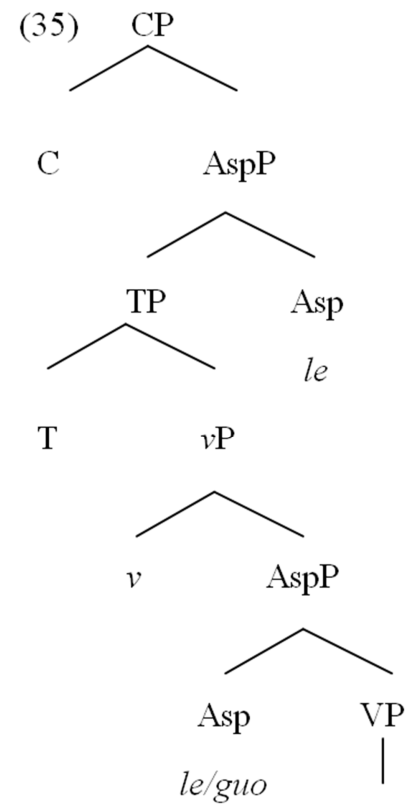

V

There are two positions for the perfective viewpoint morpheme le. The first is the head of the AspP inside vP. When generated in this position, it is realized as traditionally called verb-le. Note that guo also appears in this position and thus competes with le. This can explain why guo cannot co-occur with verb-le. The second position for le is the head of the AspP adjoined to TP. In this sentence-final position, it will form a Perfect and denote a meaning of change of state. The reason why guo cannot occur with it is the different aspectual meanings they convey. Guo is concerned with the experience the subject has while sentence-le is about the state change.

\section{References}

[1] Chao, Y.-R. (1968). A Grammar of Spoken Chinese. Berkeley and Los Angeles: University of California Press.

[2] Ching, E. \& Ching, N. (2007). 201 Mandarin Chinese Verbs. New York: Barron's Educational Series. 
[3] Chu, C.-H. C. (1998). A Discourse Grammar of Mandarin Chinese. New York: Peter Lang.

[4] Hu, Y. \& Fan, X. (1996). Dongci Yanjiu Zongshu (An Overview on the Research of Verbs in Chinese). Shanxi, China: Shanxi Higher Institution Unified Press.

[5] Leech, G. N. (2013). Meaning and the English Verb. Abingdon-on-Thames: Routledge.

[6] Li, C. N., \& Thompson, S. (1981). Mandarin Chinese. Berkeley and Los Angeles: University of California Press.

[7] Li, P. (1998). The Acquisition of Lexical and Grammatical Aspect in Chinese. First Language, 18, 311-350.

[8] Liu, Feng-his. (2012). Side by Side Chinese and English Grammar. New York: McGraw-Hill Education.

[9] Palmer, F. R. (1979). Modality and English Modals. Cambridge: Cambridge University Press.
[10] Palmer, F. R. (1974). The English Verb. Cambridge: Cambridge University Press.

[11] Smith, C. (1997). The Parameter of Aspect. Norwell, MA: Kluwer Academic Publishers.

[12] Sybesma, R. (1999). The Mandarin VP. Norwell, MA: Kluwer Academic Publishers.

[13] Wheatley, K. J. (2014). Chinese Verbs \& Essentials of Grammar. New York: McGraw-Hill Education.

[14] Wu, X.-Z. \& Liu, Feng-his. Practice Makes Perfect Basic Chinese. New York: McGraw-Hill Education.

[15] Zhu, D. (1982). Yufa Jiangyi (Lectures on Grammar). Beijing: Commercial Press. 